Pacific Journal of Mathematics

CHAIN CONDITIONS ON SUBGROUPS OF LCA GROUPS 


\title{
CHAIN CONDITIONS ON SUBGROUPS OF LCA GROUPS
}

\author{
M. A. KHAN
}

In this paper, we study ace (ascending chain condition) and dec (descending chain condition) on different types of subgroups of LCA (locally compact abelian) groups, such as open subgroups, compact subgroups, discrete subgroups, metrizable subgroups, closed divisible subgroups, proper dense subgroups. We characterize compactly generated LCA groups as the class of LCA groups whose open subgroups satisfy acc; the compactly cogenerated groups as the class of LCA groups whose discrete subgroups satisfy dec. We also show that acc and dec on the following classes of subgroups are pairwise equivalent: (a) closed subgroups (b) closed totally disconnected subgroups (c) closed $\sigma$-compact subgroups (d) closed metrizable subgroups. We also obtain a characterization of those LCA groups which contain no proper dense subgroups.

1. Introduction. In the category of abelian groups, various classes of groups can be characterized in terms of ascending or descending chain conditions on appropriate sets of their subgroups. For example, an abelian group $G$ is finitely generated (cogenerated) iff the subgroups of $G$ satisfy the ascending (respectively descending) chain condition; $G$ has finite rank iff the pure subgroups of $G$ satisfy the ascending (or equivalently descending) chain condition; the $p$-group $G$ is reduced iff its cyclic subgroups satisfy the ascending chain condition (see [4]). The aim of this paper is to obtain similar characterizations of locally compact abelian (LCA) topological groups. To be precise, we shall say that the set of subgroups with a property $P$ of an LCA group $G$, satisfy the ascending chain condition or the maximum condition or acc provided every ascending sequence of subgroups with property $P$ is stationary after a finite number of steps. There is a similar definition for the descending chain condition or the minimum condition or dec.

Throughout, all groups are assumed to be LCA Hausdorff topological groups. We shall mention the circle group $T$, the real numbers $R$, the integers $Z$, the rationals $Q$, the quasicyclic groups $Z\left(p^{\infty}\right)$, the $p$-adic integers $J_{p}$ and the $p$-adic numbers $F_{p}$. $\hat{G}$ denotes the dual groups of $G$. For any LCA group $G, T(G), D(G), B(G), S(G)$ and $G_{0}$ denote the maximal torsion subgroup of $G$, the maximal divisible subgroup of $G$, the subgroup of compact elements of $G$, the socle of $G$ and the component of the neutral element of $G$ respectively. A continuous homomorphism $f$ with domain $G$ is pro- 
per if $f$ is an open map onto $f(G)$. " $\simeq$ " denotes topological isomorphism. For other notation and terminology, we follow [4] and [5].

2. Open and compact subgroups. In this section, we study acc and dec on open subgroups and compact subgroups of LCA groups.

THEOREM 2.1. The following are equivalent for an LCA group G:

(i) $G$ is compactly generated.

(ii) The open subgroups of $G$ satisfy acc.

(iii) $\hat{G}$ has no small subgroups.

(iv) The compact subgroups of $\hat{G}$ satisfy dec.

Proof. Assume (i) and let $A_{1} \leqq A_{2} \leqq A_{3} \cdots$ be an ascending chain of open subgroups of $G$. Let $f: G \rightarrow G / A_{1}$ be the natural homomorphism onto the discrete group $G / A_{1}$. Let $G$ be generated by an open set $U$ such that $\bar{U}$ is compact. Then $G / A_{1}$ is generated by the finite set $f(\bar{U})$, so that it satisfies acc on its subgroups. It follows that the given chain of subgroups is also stationary after a finite number of steps, so that (i) $\Rightarrow$ (ii). Next assume (ii) and let $H$ be an open compactly generated subgroup of $G$ [5, 5.14], generated by a compact subset $B$. (ii) implies that $G / H$ is finitely generated. Let $A$ be a finite set in $G$ such that the image of $A$ in $G / H$ generates it. Then $A \cup B$ is a compact set generating $G$, so (ii) $\Rightarrow$ (i). (ii) $\Leftrightarrow$ (iv) is a consequence of duality. Finally, (i) $\Leftrightarrow$ (iii) because the class of compactly generated LCA groups is dual to the class of LCA groups without small subgroups [6, Corollary 1, Theorem 2.5]. This completes the proof.

THEOREM 2.2. The following are equivalent for an LCA group $G$ :

(i) The open subgroups of $G$ satisfy dec.

(ii) $G \simeq R^{n} \times M \times D$ where $M$ is compact and connected, and $D$ is discrete and finitely cogenerated.

(iii) The compact subgroups of $\hat{G}$ satisfy acc.

Proof. (i) $\Leftrightarrow$ (iii) follows by duality. Next, assume (i). Since $G_{0}$ is the intersection of all open subgroups of $G$, it is an easy consequence of condition (i) that $G_{0}$ is itself open. Hence, $G \simeq G_{0} \times$ $D$, where $D$ is discrete and its subgroups satisfy dec, so that $D$ is finitely cogenerated. This proves (ii). Conversely assume (ii). We note that $G_{0}$ is open, that every open 'subgroup of $G$ contains $G_{0}$, and $G / G_{0}$ is finitely cogenerated. Hence, by Theorem 25.1 of [4], the open subgroups of $G$ satisfy dcc. Thus (ii) $\Rightarrow(\mathrm{i})$, which completes the proof. 
In the next proposition, we show that acc on all compact subgroups of $G$ is equivalent to acc only on those closed subgroups every one of whose elements is compact.

Proposition 2.1. The following conditions on an LCA group $G$ are equivalent:

(i) The compact subgroups of $G$ satisfy acc.

(ii) The closed subgroups of $G$ with only compact elements satisfy acc.

Proof. That (ii) implies (i) is obvious. We assume (i), which implies that every closed subgroup with only compact elements is actually compact, so that (i) implies (ii).

Next, we characterize those LCA groups whose closed subgroups with only compact elements satisfy dcc.

Proposition 2.2. The following conditions on LCA group $G$ are equivalent:

(i) The closed subgroups of $G$ with only compact elements satisfy dec.

(ii) $G \simeq R^{n} \times T^{m} \times F \times H$, where $m, n$ are nonnegative integers, $F$ is discrete and finitely cogenerated and $H$ is discrete and torsionfree.

Proof. Assume (i). Since compact subgroups of $G$ also satisfy dcc, $G \simeq R^{n} \times T^{m} \times D$, where $D$ is discrete [Theorem 2.1]. Since closed torsion subgroups of $G$ satisfy dcc, $T(D)$ is finitely cogenerated and $D \simeq T(D) \times(D / T(D))$ by [4, Corollary 27.6]. This proves (ii). Next, assume (ii). Let $H$ be any closed subgroup with only compact elements. Then $H$ is isomorphic to a closed subgroup of a group of the form $T^{m} \times F$, whence the result follows from 2.1 of [1].

REMARK 2.1. The compact subgroups of $G$ satisfy acc iff the closed subgroups of $G$ with only compact elements satisfy acc [Proposition 2.1]. However, this equivalence does not hold in case of dce [compare Theorem 2.1 and Proposition 2.2].

3. Closed subgroups. To obtain a suitable characterization of compactly cogenerated LCA groups, D. L. Armacost studied acc and dec on closed subgroups of LCA groups [1, Theorem 2.1 and Corollary 2.1]. We shall show that acc (dcc) on all closed subgroups of an LCA group is equivalent to acc (dec) on any one of the following:

(a) totally disconnected, closed subgroups [Theorem 3.1]. 
(b) closed $\sigma$-compact subgroups [Theorem 3.2].

(c) closed metrizable subgroups [Propositions 4.2 and 4.3].

THEOREM 3.1. The following are equivalent for an LCA group $G$ :

(i) The closed subgroups of $G$ satisfy acc.

(ii) The closed, totally disconnected subgroups of $G$ satisfy acc.

(iii) The closed $\sigma$-compact subgroups of $G$ satisfy acc.

Proof. (i) implies (ii) is obvious. We assume (ii). Then $G_{0}$ must be compact. Since $T\left(G_{0}\right)$ is divisible and its closed subgroups satisfy acc, $T\left(G_{0}\right)$ must be trivial. Since $\hat{Q}$ contains a copy of the totally disconnected group $\Pi_{p} J_{p}$, where $p$ varies over all primes, (ii) implies that $G_{0}$ itself must be trivial. This proves (i). Since (i) $\Rightarrow$ (iii) is clear, we prove that (iii) implies (i). By Theorem 2.2 (iii) and duality $G \simeq R^{n} \times L \times M \times N$, where $L$ is discrete and torsion-free, $M$ is a finite group and $N$ is the product of finitely many $p$-adic integer groups. Since a discrete group is $\sigma$-compact iff it is countable, condition (iii) implies that $n$ must be zero. Also a little reflection shows that $L$ is free of finite rank. Hence, $G$ is the direct sum of a discrete finitely generated group, and a product of finitely many $p$-adic integer groups. This proves (i) $[1$, Corollary 2.1].

THEOREM 3.2. The following are equivalent for an LCA group G:

(i) The closed subgroups of $G$ satisfy dec.

(ii) The closed, totally disconnected subgroups of G satisfy dcc.

(iii) The closed $\sigma$-compact subgroups of $G$ satisfy dcc.

Proof. (i) implies (ii) is obvious. We shall prove that (ii) implies (i). Since the discrete subgroups of $R^{n}$ do not satisfy dec, $G_{0}$ is compact. Also (ii) implies that every torsion homomorphic image of the discrete torsion-free group $L=\left(G_{0}\right)^{\wedge}$ is finite. Let $H$ be the subgroup of $L$ generated by a maximal independent set. Then $L / H$ is finite, say of order $n$, and $n L \subseteq H$. Hence, $n L$ is free abelian. But $L \simeq n L$, so $L$ is also a free abelian group. If $L$ were of infinite rank, $L / m L(m>1)$ would be an infinite torsion group. Hence, $L$ is a free abelian group of finite rank, so that $\hat{L} \simeq T^{n}$ for some nonnegative integer $n$. Then $G \simeq T^{n} \times M$, where $M$ is totally disconnected [5, 25.31]. Let $K$ be a compact, open subgroup of $M$. By (ii) and Theorem 2.1, $K$ is discrete and, therefore, $M$ is discrete and finitely cogenerated. By Proposition 2.2, the closed subgroups 
of $G$ satisfy dec, so (ii) $\Rightarrow$ (i). Finally, since (i) $\Rightarrow$ (iii) is obvious, we prove that (iii) $\rightarrow$ (i). By Theorem 2.1 (iv), $G \simeq R^{n} \times T^{m} \times D$, where $m, n$ are nonnegative integers and $D$ is discrete. By (iii), $n=0$ and $D$ is a discrete torsion group. Let $P$ be the elementary subgroup of $D$. By (iii), the countable subgroups of $P$ satisfy dec. Hence, $P$ has only a finite number of distinct $p$-components, each of which is finite. As an essential extension of a finite group, $D$ must be finitely cogenerated $[4,25.1]$. This proves (i), and the proof of the theorem is complete.

4. Discrete subgroups. Compactly cogenerated LCA groups were defined and studied by D.L. Armacost in [1]. We showed in Theorem 2.1 that compactly generated LCA groups are characterized by acc on their open subgroups. In Theorem 4.1 below, we shall show that compactly cogenerated LCA groups are characterized by dec on their discrete subgroups.

THEOREM 4.1. The following are equivalent for an LCA group G:

(i) $G$ is compactly cogenerated.

(ii) The discrete subgroups of $G$ satisfy dec.

Proof. Assume (i) and let $H$ be a nonzero discrete subgroup of $G$. Then $L=H \cap \overline{S(G)}$ is a nonzero finite subgroup of $H$, and every nonzero subgroup of $H$ has a nontrivial intersection with $L$. [1, Theorem 3.1]. Hence, $H$ is finitely cogenerated. It follows that the discrete subgroups of $G$ 'satisfy dec [4, 25.1]. Next, assume (ii). We shall prove that every element of $G$ is compact and $\overline{S(G)}$ is compact. This will establish that $G$ is compactly cogenerated [1, Theorem 3.1]. The first assertion is obvious; $G$ cannot have a discrete, infinite cyclic subgroup. We show now that $\overline{S(G)}$ is compact. Since $S(G)$ is an elementary group, every subgroup of $S(G)$ is an algebraic direct summand of $S(G)[4$, p. 89]. Let $M$ be a compact, open subgroup of $G$. Then $S(G)=M \cap S(G)+K$, where $K$ is a subgroup of $S(G)$ such that $M \cap S(G) \cap K=\{0\}$. Clearly $M \cap K$ is trivial, so $K$ is discrete. Hence, $K$ is finite by condition (ii). Now $S(G) \subseteq \overline{M \cap S(G)}+K$, a compact subgroup. Hence, $\overline{S(G)}$ is compact, and the proof is complete.

We show next that it is the discrete subgroups of an LCA group which determine whether it is compactly cogenerated or not.

CoROLLARY 4.1. An LCA group $G$ is compactly cogenerated iff every proper discrete subgroup of $G$ is finitely cogenerated.

Proof. Let $G$ be compactly cogenerated. By Theorem 4.1, every 
proper discrete subgroup is finitely cogenerated. Conversely, let every proper discrete subgroup be finitely cogenerated. Take a chain $A_{1} \supseteqq A_{2} \supseteqq, \cdots$, of discrete subgroups of $G$. If each $A_{i}=G$, the chain is stationary. Otherwise, some $A_{i}$ is proper, hence finitely cogenerated, so it satisfies dcc. Hence, $G$ is compactly cogenerated.

Using Theorem 4.1 and Corollary 4.1, we get the following stronger version of Proposition 6.3 of [1].

Corollary 4.2. Let $G$ be an LCA group.

(i) If $G$ is compactly cogenerated, then every closed subgroup of $G$ is compactly cogenerated.

(ii) If every proper discrete subgroup of $G$ is finitely cogenerated then $G$ is compactly cogenerated.

It was pointed out in [1] that the quotient of a compactly cogenerated group by a closed subgroup need not be compactly cogenerated. We show below that the quotients by discrete subgroups behave nicely.

CoROLlary 4.3. Let $G$ be compactly cogenerated and let $H$ be a discrete subgroup. Then $G / H$ is compactly cogenerated.

Proof. Let $L / H$ be a discrete subgroup of $G / H$. Then $H$ is an open subgroup of the LCA group $L$. Since $H$ is discrete, $L$ is also discrete. Hence, $L$ is finitely cogenerated and consequently $L / H$ is finitely cogenerated. Corollary 4.1 completes the proof.

Before passing on to the maximum condition on discrete subgroups, we use Theorem 4.1 to investigate the minimum condition on closed, metrizable subgroups of LCA groups.

Proposition 4.1. The closed metrizable subgroups of an LCA group $G$ satisfy dec iff the closed subgroups of $G$ satisfy dec.

Proof. We assume the minimum condition on closed metrizable subgroups. Since discrete subgroups are metrizable, $G$ is compactly cogenerated. In particular, every element of $G$ is compact and $G_{c}$ is compact. By dec on metrizable subgroups of $G_{0}$, it follows that every countable homomorphic image of the discrete, torsion-free group $\left(G_{0}\right)^{\wedge}=L$, say, is finitely generated. Let $H$ be a subgroup of $L$ such that $L / H$ is a torsion group. If $L / H$ were infinite, there would be a subgroup $K$ of $L$, containing $H$, such that $(L / H) /(K / H)$ is a countably infinite torsion group [5, 16.13(c)]. But this would imply that $L / K$ is a finitely generated infinite torsion group. Hence, 
every torsion homomorphic image of $L$ is finite. It then follows, as in the proof of Theorem 3.2, that $L$ is a discrete free group of finite rank. Hence, $\hat{L} \simeq T^{n}$ for some nonnegative integer $n$. Then $G \simeq T^{n} \times M$, where $M$ is totally disconnected. By the minimum condition and [5, 25.16], every compact, monothetic subgroup of $M$ is finite. Hence, $M$ is a torsion group. By [1, 6.1], $M$ is the topological direct sum of finitely many $p$-groups each having compact socle. Let $S_{p}$ denote the compact socle of the $p$-component of $M$. Since $S_{p}$ is the compact product of groups $Z_{p}$, the minimum condition implies that $S_{p}$ is finite. Hence, each $p$-component of $M$ is discrete and finitely cogenerated. We conclude from Theorem 2.1 of [1] that the closed subgroups of $G$ satisfy dcc. Hence, $M$ itself is discrete and finitely cogenerated. As the converse is obvious, the proof is complete.

Next we investigate the maximum condition on discrete subgroups. We first prove a lemma.

LeMMA 4.1. Let $G$ be an LCA group such that $T(G)$ is closed. Then $G_{0}$ is a topological direct summand of $G$ and $G \simeq R^{m} \times M \times N$, where $M$ is compact, connected, torsion-free group, and $N$ is a totally disconnected group such that $T(N)$ is closed.

Proof: We can assume, without loss of generality, that $G$ has a compact, open subgroup $H$, and $G_{0}$ is compact. Let $H \cap T(G)=F$. Then by [5, Theorem 25.9], $F$ is a compact, torsion group of bounded order. Also $G_{0} \cap T(G)$ is a divisible group of bounded order, so it must be trivial. Since $H /\left(G_{0}+F\right)$ is compact and torsion-free, duality shows that $G_{0}+F$ is a topological direct summand of $H$. Hence, by $[5,6.12], H=G_{0}+F+K$, for some compact subgroup $K$, is a decomposition of $H$ into a topological direct sum, so that $G_{0}$ is a topological direct summand of $H$. Hence, there is a continuous epimorphism $f: H \rightarrow G_{0}$ such that $f \mid G_{0}$ is the identity map of $G_{0}$. Since $H$ is open and $G_{0}$ is divisible, $f$ extends to a continuous emimorphism $F: G \rightarrow G_{0}$ such that $F\left|G_{0}=f\right| G_{0}$. Hence, $G_{0}$ is a topological direct summand of $G$. Since $G_{0} \cap T(G)$ is trivial, it is clear that the structure of $G$ is as stated above.

THEOREM 4.2. The discrete subgroups of LCA group $G$ satisfy acc iff $G \simeq G_{0} \times N$, where $G_{0}$ is compact, connected, torsion-free, $N$ is a totally disconnected group, $T(N)$ is finite, and there is a nonnegative integer $n$ such that every $B(N)$-high subgroup of $N$ is a free abelian group of rank $n$.

Proof. Assume acc on discrete subgroups. Clearly $R^{n}$ is not a 
topological direct summand of $G$, and $T(G)$ is finite. Hence, by Lemma 4.1, $G \simeq G_{0} \times N$, where $G_{0}$ is compact, connected and torsionfree, and $T(N)$ is finite. Clearly $B(N)$ is an open, pure subgroup of $N$ such that $N / B(N)$ is torsion-free. Also, any $B(N)$-high subgroup of $N$ is discrete, and free of finite rank because of the maximum condition on discrete subgroups. Let $C$ be a subgroup of $N / B(N)$ generated by a maximal independent subset. Let $L$ be a subgroup of $N$ such that $L / B(N) \simeq C$. Since $B(N)$ is a pure subgroup of $L, L=B(N) \times H$ [4, Theorem 28.2], where $H$ is discrete and free of finite rank, say $n$. Then $C$ is of rank $n$. It is now obvious that every $B(N)$-high subgroup of $N$ is free of rank $n$. For the converse, we observe that any discrete subgroup of $G$ is of the form $D \times E$, where $D$ is a subgroup of $T(N)$ and $E$ is a free abelian group with rank $E \leqq n$, such that $B(N) \cap E$ is trivial. It is then clear that the discrete subgroups of $G$ satisfy the maximum condition. This completes the proof.

We now use Theorem 4.2 to investigate maximum condition on closed, metrizable subgroups of LCA group.

Proposition 4.2. The closed metrizable subgroups of an LCA group $G$ satisfy acc iff the closed subgroups of $G$ satisfy acc.

Proof. Assume the maximum condition on closed metrizable subgroups. Since discrete subgroups are metrizable and the closed (metrizable) subgroups of $\hat{Q}$ do not satisfy acc, $G$ is a totally disconnected group such that $T(G)$ is finite and every $B(G)$-high subgroup of $G$ is a discrete free subgroup of fixed finite rank [Theorem 4.2]. Let $H_{1}, H_{2}$ be (compact metrizable) monothetic subgroups of $B(G)$. It is easy to show that $H_{1}+H_{2}$ is also a compact, metric group. By acc, it follows that $B(G)$ is compact. With the aid of duality, one shows easily that $B(G)=T(G) \times M$, where $M$ is a product of finitely many $p$-adic integer groups. Also, $B(G)$ is a pure, compact, open subgroup of $G$, so that $G=T(G) \times M \times N$, where $N$ is a discrete, free subgroup of finite rank. Hence, the closed subgroups of $G$ satisfy acc. As the converse is obvious, the proof is complete.

5. Nonclosed subgroups and proper dense subgroups. In this section, we study acc and dec on nonclosed subgroups, and proper, dense subgroups of LCA groups. We would like to point out that there is an interesting discussion of LCA groups without proper, dense subgroups in [7]. First, we prove a number of lemmas.

LEMmA 5.1. Let $G$ be a nondiscrete, compact abelian group. 
Then the proper, dense subgroups of $G$ satisfy neither acc nor dec.

Proof. $\hat{G}$ is discrete and infinite. Let $H$ by a countably infinite subgroup of $\hat{G}$. Then there exists a proper, continuous epimorphism of $G$ onto the compact, metric group $\hat{H}$. Since $\hat{H}$ is uncountable and contains a countable, dense, proper subgroup, it is clear that the dense, proper subgroups of $\hat{H}$ satisfy neither acc nor dec. The proper epimorphism of $G$ onto $\hat{H}$ ensures that the same is true of proper, dense subgroups of $G$.

LEMma 5.2. Let $G$ be an LCA group which is not totally disconnected. Then the proper, dense subgroups of G satisfy neither acc nor dec.

Proof. Since $G$ is not totally disconnected, $\hat{G}$ contains a copy of $Z$. Hence, there is a closed subgroup $H$ of $G$ such that $G / H \simeq T$. The assertion about proper, dense subgroups is now obvious.

LEMma 5.3. Let $G$ be a nondiscrete, totally disconnected, divisible LCA group such that $B(G)$ has an element of infinite order. Then the proper, dense subgroups of $G$ satisfy neither acc nor dec.

Proof. Since $G$ is divisible and $G / B(G)$ is torsion-free, $B(G)$ is also divisible. Since $B(G)$ is also open, we have $G=B(G) \times K$, where $K$ is a discrete, divisible, torsion-free group. We shall show that $B(G)$ satisfies neither acc nor dec on proper, dense subgroups. Since $B(G)$ is not a torsion group, it contains a copy of $Q$ as an abstract group. By [3], $\bar{Q}$ is divisible. Since $\bar{Q}$ is uncountable and $Q$ is dense in it, there exists a strictly ascending (descending) sequence of proper subgroups $A_{i}\left(B_{i}\right)$ of $\bar{Q}$ such that each $A_{i}\left(B_{i}\right)$ is dense in $\bar{Q}$. Since $\bar{Q}$ is divisible, there is a subgroup $N$ of $B(G)$ such that $B(G)=\bar{Q}+N$ and $\bar{Q} \cap N$ is trivial. As $\overline{A_{i}+N} \supseteqq \bar{A}_{i}+\bar{N}=B(G)$ and $\overline{B_{i}+N} \supseteq \bar{B}_{i}+\bar{N}=B(G)$, it is clear that the groups $A_{i}+N$ (respectively $B_{i}+N$ ) provide a strictly ascending (descending) sequence of proper, dense subgroups of $G$.

REMARKS 5.1. Under the hypothesis of Lemma 5.3, there is a subgroup $L$ of $G$ such that $L$ is dense in $G, L$ contains a copy of $Q$ and $G / L$ is uncountable. In fact, such a group is $L=Q+N+K$, in the notation of the proof of Lemma 5.3.

LEMMA 5.4. Let $G$ be a nondiscrete, totally disconnected LCA group such that $B(G)$ has an element of infinite order. Then the proper, dense subgroups of $G$ eatisfy neither acc nor dec. 
Proof. Let $G^{*}$ be a minimal divisible extention of $G$, topologized so that $G$ is an open subgroup. Then $G^{*}$ will satisfy the hypotheses of Lemma 5.3. Let $L$ be a dense subgroup of $G^{*}$ such that $G^{*} / L$ is uncountable [see Remark 5.1]. Then $G \cap L$ is a proper, dense subgroup of $G$ and $G^{*} / L=(G+L) / L \approx G / G \cap L$ is uncountable. Since the proper subgroups of $G / G \cap L$ satisfy neither acc nor dcc, the same is true of dense, proper subgroups of $G$ containing $G \cap L$. This completes the proof.

Lemma 5.5. Let $G$ be a totally disconnected LCA group such that $B(G)=T(G)$, and let $H$ be a compact, open subgroup (necessarily of bounded order) of $G$ such that $n H=0$. Then every dense subgroup of $G$ contains $n G$.

Proof. Let $K$ be a dense subgroup of $G$ and let $n g \in n G$. Since $(g+H) \cap K$ is not empty, there is an element $g+h$ in $K$, where $h \in H$. As $n(g+h)=n g+n h=n g$, we see that $n g \in K$.

Lemma 5.6. Let $G$ be a totally disconnected, divisible LCA group such that $B(G)=T(G)$. Then $G$ contains no proper, dense subgroup.

Proof. Let $H$ be a compact, open subgroup satisfying $n H=0$. Since $n G=G$, Lemma 5.5 completes the proof.

Next, we establish a necessary and sufficient condition in order that an LCA group may contain no proper, dense subgroup.

Proposition 5.1. Let $G$ be a nondiscrete LCA group. Then $G$ has no proper, dense subgroup iff

(a) $G$ is totally disconnected.

(b) $B(G)=T(G)$.

(c) For every prime $p, p G$ is an open subgroup of $G$.

Proof. Assume $G$ satisfies (a), (b) and (c). Let $H$ be a compact, open subgroup such that $n H=\{0\}$ and let $K$ be a proper, dense subgroup of $G$. Since $n G \subseteq K$ (Lemma 5.5), $n G$ is a proper subgroup of $G$. Clearly, $G / K$ is a group of bounded order. Hence, there is a subgroup $L$ of $G$, containing $K$ such that $L$ is of prime index in $G$, say $p$. But $p G$ is open, so $L$ is open, contradicting $\bar{K}=$ $G$. Hence, $G$ has no proper, dense subgroup.

Conversely, assume $G$ has no proper, dense subgroup. Then (a) is satisfied [Lemma 5.2]. If $B(G)$ is not a torsion group, let $D$ be 
the minimial divisible extension of $G$ with the usual topology. Then $D$ has proper, dense subgroups [Lemma 5.3], and so does $G$. Hence, $G$ satisfies (b). Next, suppose $p G \neq G$ for some prime $p$. Then every subgroup of index $p$ (necessarily a maximal subgroup of $G$ ) must be closed. By [4, p. 18], as $p G$ is the intersection of all such subgroups, $p G$ is closed. Now $G / p G$ is the topological direct sum of a discrete group and a compact group, say $M[5,25.29]$. Since $G$ has no proper, dense subgroup, $M$ has to be a finite group [Lemma 5.1]. Hence, $G / p G$ is discrete, so $p G$ is open, which shows that (c) is satisfied. This completes the proof.

CoRollary 5.1. A nondiscrete LCA group $G$ has no proper, dense subgroups iff

(a) $T(G)$ is open.

(b) $p G$ is open, for every prime $p$.

Proof. (a) and (b) of Proposition 5.1 imply that $B(G)$ is open, and hence, $T(G)$ is open. Conversely, $T(G)$ is open implies that $G$ is totally disconnected and $T(G)=B(G)$.

COROLLARY 5.2. If a nondiscrete LCA group $G$ has no proper, dense subgroup, then $\widehat{G}$ is compactly cogenerated and every continuous character of $\hat{G}$ is proper.

Proof. Assume $G$ has no proper, dense subgroup. We shall prove first that $\hat{G}$ is compactly cogenerated. Since $G$ is totally disconnected [Proposition 5.1], every element of $\hat{G}$ is compact. Next, we show that the intersection of all the subgroups $p G$ is open. Let $H$ be a compact, open subgroup with $n H=0$. For any prime $q$ which is prime to $n, H \subseteq q G$, Hence, $H \subseteq \cap q G$, where $q$ varies over all those primes which are prime to $n$. It follows that $\cap q G$ is open. Since only finitely many primes divide $n$, it is clear that $\cap p G$, as $p$ varies over all primes, is an open subgroup of $G$. Using these facts and duality, we have that every element of $\hat{G}$ is compact and $\widehat{S(\widehat{G})}$ is compact. Hence, $\hat{G}$ is compactly cogenerated. Next, we note that a continuous character $\widehat{G} \rightarrow T$ is proper iff the adjoint $Z \rightarrow G$ is proper [6, Prop. 2.2]. Hence, a continuous character $g$ of $\widehat{G}$ is proper iff either $g$ is of finite order or $g$ generates a discrete, infinite cyclic subgroup of $G$. It then follows by Proposition 5.1 (b) that every continuous character of $\hat{G}$ is proper.

CoROLlaRY 5.3. If an LCA group $G$ has no proper, dense subgroup, then every neat, closed subgroup of $G$ (in particular, $T(G))$ has the same property. 
Proof. Let $H$ be a neat, closed subgroup. Then $H$ is totally disconnected, $T(H)=B(H)$, and since $p H=H \cap p G, p H$ is open in $H$ for every prime $p$. The assertion follows by Proposition 5.1.

Corollary 5.4. A divisible LCA group $G$ has no proper, dense subgroup iff $G \simeq L \times M$, where $L$ is a divisible, torsion LCA group, and $M$ is discrete, divisible and torsion-free.

Proof. If $G$ has no proper, dense subgroup, then $T(G)$ is open. As $T(G)$ is also divisible, it is a topological direct summand. Taking $T(G)=L$, the assertion follows. Conversely, assume $G$ has a proper, dense subgroup. Then $L$, being an open subgroup, also has a proper, dense subgroup, contradicting Proposition 5.1. Hence, $G$ can have no proper, dense subgroup.

CoROLLARY 5.5. Let $G$ be an LCA group with no proper, dense subgroup. Then the closure of every nonclosed, divisible subgroup is nondivisible,

Proof. Let $H$ be a nonclosed divisible subgroup. If $\bar{H}$ were divisible, it would be a pure subgroup of $G$. Hence, by Corollary 5.3, $\bar{H}$ must be nondivisible.

In the remainder of this section, we shall prove our results on acc and dec on nonclosed and proper, dense subgroups.

THEOREM 5.1. Let $G$ be a nondiscrete LCA group. Then the nonclosed subgroups of $G$ satisfy neither acc nor dec.

Proof. If $G$ is not totally disconnected, the assertion follows from Lemma 5.2. If $G$ is totally disconnected, $G$ has a compact, open subgroup and Lemma 5.1 completes the proof.

THEOREM 5.2. Let $G$ be a nondiscrete LCA group such that either $G$ is not totally disconnected or $G$ is totally disconnected and $B(G)$ has an element of infinite order, then the proper, dense subgroups of $G$ satisfy neither acc nor dec.

Proof. If $G$ is not totally disconnected, the assertion is a consequence of Lemma 5.2. If $G$ is totally disconnected and $B(G)$ has an element of infinite order, Lemma 5.3 completes the proof.

The last theorem says nothing about those totally disconnected LCA groups for which $T(G)=B(G)$. In fact, we have not succeeded 
in obtaining a satisfactory answer in this case. However, the next proposition deals with this case under some additional hypotheses.

Proposition 5.2. Let $G$ be a nondiscrete, totally disconnected LCA group such that $T(G)=B(G)$.

(a) If $p G$ is open for every prime $p$, then the proper, dense subgroups of $G$ satisfy both acc and dec (vacuously).

(b) If condition (a) given above is not satisfied, but $\overline{p G}=G$ for every prime $p$, then the dense, proper subgroups of $G$ satisfy acc (dcc) iff $G / D(G)$ is finitely generated (finite).

Proof. (a) follows from Proposition 5.1. For (b), we first observe that $G$ is not divisible, but since $\hat{G}$ is torsion-free, $G$ contains a dense, divisible subgroup [8]. Let $H$ be a compact, open subgroup with $n H=0$. Then by Lemma 5.5, every proper, dense subgroup contains $n G$. On the other hand, $n G$ contains the proper, dense subgroup $D(G)$. It follows that every proper, dense subgroup of $G$ contains $D(G)$. The assertion in (b) is now obvious.

6. Divisible subgroups. In order to study LCA groups whose divisible subgroups satisfy acc or dcc, we prove two lemmas.

LEMma 6.1. A connected, LCA group $G$ is finite-dimensional iff the closed divisible subgroups of $G$ satisfy acc (or equivalently, dec).

Proof. Since $R^{n}$ is finite-dimensional and its closed divisible subgroups satisfy acc and dcc, we suppose $G$ is compact. Then $\hat{G}$ is discrete and torsion-free. Since the pure subgroups of $\hat{G}$ satisfy acc (or dec) iff $\hat{G}$ is of finite rank, and dimension $G=\operatorname{rank}$ of $\hat{G}$ $[5,24.8]$, the assertion about $G$ follows.

LEMMA 6.2. If the closed divisible subgroups of an LCA group satisfy acc, then $D(G)$ is a closed subgroup.

Proof. Let $H_{1}$ be a divisible subgroup of finite rank. Then $\bar{H}_{1}$ is divisible [3]. If $\bar{H}_{1}$ is not equal to $D(G)$, there is a divisible subgroup $H_{2}$ of finite rank such that the divisible subgroup $\bar{H}_{2}$ contains $\bar{H}_{1}$ properly. The chain condition then ensures that $D(G)$ is closed.

Following [1], we say that an LCA group is indecomposable iff it cannot be written as the topological direct sum of two of its proper closed subgroups; otherwise it is decomposable. Then an 
indecomposable divisible LCA group is either compact connected, or is topologically isomorphic to either $R$ or $Q$ or $Z\left(p^{\infty}\right)$ or the $p$-adic number group $F_{p}$ [1, Theorem 6.1]. We shall say that a divisible LCA group is finitely decomposable if it can be written as the direct sum of finitely many indecomposable divisible LCA groups so that each compact connected summand is finite-dimensional. We also recall that an LCA group is torsion-free iff its dual group is densely divisible i.e., contains a dense, divisible subgroup [8, 5.2].

Following these observations, we next prove a number of lemmas to arrive at the main results.

Lemma 6.3. Let $G$ be a finitely decomposable, divisible LCA group. Then the closure of every divisible subgroup of $G$ is divisible.

Proof. We first note that $G$ is $\sigma$-compact. Let $H$ be a divisible subgroup of $G$ and $n$ a natural number. Then $G(n)$, the closed subgroup of those elements of $G$ whose order divides $n$, is a fully invariant subgroup of $G$. Hence, $G(n)=(G(n) \cap C) \times(G(n) \cap P)$, where $C$ is the compact, connected summand of $G$ and $P$ is a finite direct sum of quasicyclic groups [4, Lemma 9.3 and 5, Theorem 6.12]. Clearly, $G(n)$ is compact. As $G$ is $\sigma$-compact and divisible, multiplication by $n$ is an open, continuous epimorphism of $G$ [5, 5.29]. Hence, $n \bar{H}=n(\bar{H}+G(n))$ is a closed subgroup of $G$. But $n H \subseteq n \bar{H}$, so $\bar{H}=\overline{n H} \subseteq n \bar{H}$. Hence, $n \bar{H}=\bar{H}$, and $\bar{H}$ is divisible.

Corollary 6.1. Let $G$ be a finitely decomposable, divisible LCA group. Then a closed subgroup of $G$ is divisible iff its annihilator is a pure subgroup of $\hat{G}$.

Proof. As $\hat{G}$ is torsion-free, the result follows by the last lemma and [8, 5.2].

LEMMA 6.4. The closed, pure subgroups of an LCA group $G$ of the form $R^{n} \times M$, where $M$ is a finite product of p-adic number groups, satisfy both acc and dec.

Proof. Let $H$ be a closed, pure subgroup of $G$, then $H$ is divisible. We claim that $H=\left(R^{n} \cap H\right) \times(M \cap H)$. To show this, first we note that $G_{0}=R^{n}, B(G)=M, H_{0}=R^{n} \cap H$ and $B(H)=M \cap$ $H$. As $H_{0} \times B(H)$ is an open divisible subgroup of $H$, we must have $H=H_{0} \times B(H)$ algebraically, for if not, then $H$ would contain 
a discrete copy of $Q$, which is impossible. Theorem 6.12 of [5] then completes the proof of the claim. This reduces the problem to acc and dec on closed, divisible subgroups of $R^{n}$ and $M$ respectively. For $R^{n}$, it is obvious. For $M$, we observe that $M$ and its closed, divisible subgroups are minimal divisible extensions of finite products of $p$-adic integer groups [5, 25.32], which satisfy acc on their closed subgroups. This implies acc on closed, divisible subgroups of $M$. As $M$ is self-dual [5, 25.34], this in its turn implies dec on closed, divisible subgroups of $M$.

LEMMA 6.5. Let $G=R^{n} \times M \times N$, where $M$ is a discrete, torsion-free group of finite rank and $N$ is a finite product of p-adic number groups. Then the closed, pure subgroups of $G$ satisfy both acc and dec.

Proof. Let $H_{1}, H_{2}$ be closed, pure subgroups of $G$ such that $H_{1} \subseteq H_{2}$. Let $L=R^{n} \times N$. Then $L=G_{0}+B(G)$ and $H_{1} \cap L, H_{2} \cap L$ are divisible, open subgroups of $H_{1}$ and $H_{2}$ respectively. Hence, $H_{1}=\left(H_{1} \cap L\right) \times A_{1}$, and $H_{2}=\left(H_{2} \cap L\right) \times A_{2}$, where $A_{1}, A_{2}$ are discrete torsion-free groups of finite rank, and we can arrange that $A_{1} \subseteq$ $A_{2}$ [4, 21.2]. The assertion of the lemma now follows by Lemma 6.4.

LEMma 6.6. The closed, divisible subgroüps of a finitely decomposable divisible LCA group satisfy both acc and dec.

Proof. In view of Corollary 6.1, it is enough to prove that the closed pure subgroups of a group of the form $G=R^{n} \times L \times M \times$ $N \times P$, satisfy both acc and dcc, where $L$ is a discrete torsion-free group of finite rank, $M$ and $N$ are finite products of $p$-adic number groups and $p$-adic integer groups respectively, and $P$ is product of finitely many copies of $\hat{Q}$. Let $H_{i}(i=1,2,3, \cdots)$ denote an ascending (or descending) chain of closed, pure subgroups of $G$. Then $H_{i} \cap(N \times P)$ is a pure, closed subgroup of $N \times P$ for each $i$. Also $H_{i}+(N \times P)$ is a closed subgroup of $G$, because $N \times P$ is compact. Now $H_{i}+(N \times P)=(N \times P) \times\left(H_{i} \cap\left(R^{n} \times L \times M\right)\right)$, by [4, p. 38 and 5,6.12]. Since our groups are all torsion-free, it is clear that $H_{i}+(N \times P)$ is a closed, pure subgroup of $G$ for each $i$.

By duality and Corollary 6.1, it is obvious that the closed pure subgroups of $N \times P$ satisfy both acc and dec. Also by the decomposition of $H_{i}+(N \times P)$ given above and Lemma 6.5, the groups $H_{i}+(N \times P)$ are stationary after some value of $i$, whether the chain is ascending or descending. We can assume, without loss of generality, that $H_{n} \cap(N \times P)=H_{m} \cap(N \times P)$ and $H_{n}+(N \times P)=$ $H_{m}+(N \times P)$ whenever $n \geqq m$, whether the chain is ascending or 
descending. Then, for every $n \geqq m$,

$$
\begin{aligned}
H_{n} & =H_{n} \cap\left(H_{n}+N \times P\right) \\
& =H_{n} \cap\left(H_{m}+N \times P\right) \\
& =H_{m}+\left(H_{n} \cap(N \times P)\right) \text { by the modular law, } \\
& =H_{m}+\left(H_{m} \cap(N \times P)\right) \\
& =H_{m}
\end{aligned}
$$

in the ascending case. In the descending case, we get the same conclusion by interchanging $m$ and $n$. This completes the proof.

We now come to the main results of this section.

Proposition 6.1. The following are equivalent for a divisible LCA group G:

(i) The closed divisible subgroups satisfy acc.

(ii) The closed divisible subgroups satisfy dcc.

(iii) $G$ is finitely decomposable.

Proof. Assume (i) or (ii) and suppose (iii) is false. Then $G=$ $G_{1} \times G_{2}$, where at least one summand is not finitely decomposable. Proceeding in this way, it is easy to see that we get both a strictly increasing and a strictly decreasing sequence of closed divisible subgroups of $G$. Hence, (i) implies (iii) and (ii) implies (iii). That (iii) implies (i) and (ii) follows from Lemma 6.6.

CoROLlaRY 6.1. Every closed divisible subgroup of a finitely decomposable group is again finitely decomposable.

THEOREM 6.1. The following are equivalent for an LCA group G:

(i) The closed divisible subgroups satisfy acc.

(ii) The closed densely divisible subgroups satisfy acc.

(iii) The closed densely divisible subgroups satisfy dcc.

(iv) $D(G)$ is closed and finitely decomposable.

Proof. Assume (iv). Using Proposition 6.1 and Lemma 6.3, it is clear that (iv) implies (i), (ii) and (iii). Next we assume (i). By Lemma 6.2, $D(G)$ is closed. An application of Proposition 6.1 then proves (iv). Clearly (ii) implies (i). Hence, (ii) implies (iv). Finally, assume (iii). We shall prove our assertion (iv). For simplicity of notation, we suppose $D(G)$ is dense in $G$. Then $\hat{G}$ is torsion-free and $(\widehat{G})_{0}$ splits out $[5,25.30]$. By (iii) and duality, $G \simeq R^{n} \times L \times M$, where $L$ is direct sum of finitely many copies of discrete group $Q$, and $M$ is densely divisible with every element compact. We shall 
prove $M$ is divisible. Now $\hat{M}$ is a torsion-free, totally disconnected LCA group, and its closed pure subgroups satisfy acc. Let $N$ be a minimal divisible extension of $\hat{M}$ topologized in the usual manner so that $\hat{M}$ is an open subgroup. Let $A_{1}, A_{2}$ be closed divisible subgroups of $N$ such that $A_{1}$ is a proper subgroup of $A_{2}$. Then $A_{1} \cap$ $\widehat{M}, A_{2} \cap \widehat{M}$ are closed pure subgroups of $\widehat{M}$. Also $A_{1} \cap \widehat{M}$ is a proper subgroup of $A_{2} \cap \hat{M}$. Hence, every properly ascending sequence of closed divisible subgroups of $N$ provides a properly ascending sequence of closed pure subgroups of $\hat{M}$. Hence, by Prosition 6.1, $N$ is a finitely decomposable, divisible, totally disconnected torsionfree group i.e., $N$ is the direct product of finitely many copies of $Q$ and finitely many $p$-adic number groups. Then $N$ is divisible. Since $M$ is a homomorphic image of $N, M$ is divisible. Hence, $G$ is divisible and finitely decomposable. This completes the proof.

COROLLARY 6.1. The following are equivalent for a torsion-free LCA group G:

(i) The closed pure subgroup of $G$ satisfy acc.

(ii) The closed pure subgroups of $G$ satisfy dec.

(iii) $G$ is the topological direct sum of $R^{n}$, finitely many groups $Q$ and $\hat{Q}$, and finitely many p-adic integer groups and p-adic number groups.

Proof. We note that $\hat{G}$ is densely divisible, and a closed subgroup of $G$ is pure iff its annihilator in $\hat{G}$ is densely divisible. The result then follows by Theorem 6.1 and duality.

Missing from the list in Theorem 6.1 is dec on closed divisible subgroups. The following example shows that dec on closed divisible subgroups is not equivalent to any condition in Theorem 6.1. Let $G$ be the local direct product of a countably infinite number of copies of group $F_{p}$, for a fixed prime $p$, relative to the subgroups $J_{p}$. Then $G$ is a densely divisible but not divisible, self-dual LCA group [5, 25.34], but the closed divisible subgroups of $G$ satisfy dec. This example is typical of the general situation.

Proposition 6.2. The closed divisible subgroups of an LCA group $G$ satisfy dcc iff the closed divisible subgroups of $G$ are finitely decomposable.

Proof. Follows from Proposition 6.1.

Finally, I would like to thank the referee for his several very useful suggestions. 


\section{REFERENCES}

1. D. L. Armacost, Compactly cogenerated LCA groups, Pacific J. Math., 65 (1976), 1-12.

2. D. L. Armacost and W. L. Armacost, On p-thetic groups, Pacific J. Math., 41 (1972), 295-301.

3. — On Q-dense and densely divisible LCA groups, Proc. Amer. Math. Soc., 36 (1972), 301-305.

4. L. Fuchs, Infinite Abelian Groups, Vol. 1, Academic Press, New York, 1970.

5. E. Hewitt and K. Ross, Abstract Harmonic Analysis, Vol. 1, Academic Press, New York, 1963.

6. M. Moskowitz, Homological algebra in locally compact Abelian groups, Trans. Amer. Math. Soc., 127 (1967), 361-404.

7. M. Rajagoplan and H. Subrahmanian, Dense subgroups of locally compact groups, Colloq. Math., Vol. XXXV (1976), 289-292.

8. L. Robertson, Connectivity, divisibility and torsion, Trans. Amer. Math. Soc., 128 (1967), 482-505.

Received April 24, 1978 and in revised form March 16, 1979.

KUWAIT UNIVERSITY

KUWAIT 


\title{
PACIFIC JOURNAL OF MATHEMATICS
}

\section{EDITORS}

\author{
DoNALD BABBITT (Managing Editor) \\ University of California \\ Los Angeles, CA 90024 \\ HUGo RossI \\ University of Utah \\ Salt Lake City, UT 84112 \\ C. C. MOORE and ANDREW OGG \\ University of California \\ Berkeley, CA 94720
}

J. DugundJI

Department of Mathematics

University of Southern California

Los Angeles, CA 90007

R. FINN and J. Milgram

Stanford University

Stanford, CA 94305

\section{ASSOCIATE EDITORS}
E. F. BeCKenbach
B. H. NeumanN
F. WOLF
K. YoshidA

\section{SUPPORTING INSTITUTIONS}

\author{
UNIVERSITY OF BRITISH COLUMBIA \\ CALIFORNIA INSTITUTE OF TECHNOLOGY \\ UNIVERSITY OF CALIFORNIA \\ MONTANA STATE UNIVERSITY \\ UNIVERSITY OF NEVADA, RENO \\ NEW MEXICO STATE UNIVERSITY \\ OREGON STATE UNIVERSITY \\ UNIVERSITY OF OREGON
}

\author{
UNIVERSITY OF SOUTHERN CALIFORNIA \\ STANFORD UNIVERSITY \\ UNIVERSITY OF HAWAII \\ UNIVERSITY OF TOKYO \\ UNIVERSITY OF UTAH \\ WASHINGTON STATE UNIVERSITY \\ UNIVERSITY OF WASHINGTON
}

The Supporting Institutions listed above contribute to the cost of publication of this Journal, but they are not owners or publishers and have no responsibility for its content or policies.

Mathematical papers intended for publication in the Pacific Journal of Mathematics should be in typed form or offset-reproduced, (not dittoed), double spaced with large margins. Please do not use built up fractions in the text of the manuscript. However, you may use them in the displayed equations. Underline Greek letters in red, German in green, and script in blue. The first paragraph or two must be capable of being used separately as a synopsis of the entire paper. Please propose a heading for the odd numbered pages of less than 35 characters. Manuscripts, in triplicate, may be sent to any one of the editors. Please classify according to the scheme of Math. Reviews, Index to Vol. 39. Supply name and address of author to whom proofs should be sent. All other communications should be addressed to the managing editor, or Elaine Barth, University of California, Los Angeles, California, 90024.

50 reprints to each author are provided free for each article, only if page charges have been substantially paid. Additional copies may be obtained at cost in multiples of 50 .

The Pacific Journal of Mathematics is issued monthly as of January 1966. Regular subscription rate: $\$ 84.00$ a year $(6$ Vols., 12 issues). Special rato: $\$ 42.00$ a year to individual members of supporting institutions.

Subscriptions, orders for numbers issued in the last three calendar years, and changes of address shoud be sent to Pacific Journal of Mathematics, P.O. Box 969, Carmel Valley, CA 93924, U.S.A Old back numbers obtainable from Kraus Periodicals Co., Route 100, Millwood, NY 10546.

\section{PUBLISHED BY PACIFIC JOURNAL OF MATHEMATICS, A NON-PROFIT CORPORATION}

Printed at Kokusai Bunken Insatsusha (International Academic Printing Co., Ltd.). 8-8, 3-chome, Takadanobaba, Shinjuku-ku, Tokyo 160, Japan. 


\section{Pacific Journal of Mathematics}

\section{Vol. 86, No. 2 December, 1980}

Graham Donald Allen, David Alan Legg and Joseph Dinneen Ward, Hermitian

liftings in Orlicz sequence spaces ............................... 379

George Bachman and Alan Sultan, On regular extensions of measures ........ 389

Bruce Alan Barnes, Representations Naimark-related to $*$-representations; a

correction: "When is a representation of a Banach $*$-algebra

Naimark-related to a $*$-representation?" ........................ 397

Earl Robert Berkson, One-parameter semigroups of isometries into $H^{p} \ldots \ldots .403$

M. Brodmann, Piecewise catenarian and going between rings ............ 415

Joe Peter Buhler, A note on tamely ramified polynomials ............... 421

William Lee Bynum, Normal structure coefficients for Banach spaces ........ 427

Lung O. Chung, Biharmonic and polyharmonic principal functions ......... 437

Vladimir Drobot and S. McDonald, Approximation properties of polynomials

with bounded integer coefficients .............................

Giora Dula and Elyahu Katz, Recursion formulas for the homology of

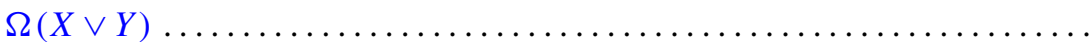

John A. Ernest, The computation of the generalized spectrum of certain Toeplitz operators ...................................... 463

Kenneth R. Goodearl and Thomas Benny Rushing, Direct limit groups and the

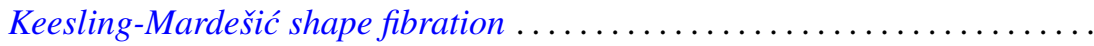

Raymond Heitmann and Stephen Joseph McAdam, Good chains with bad contractions

Patricia Jones and Steve Chong Hong Ligh, Finite hereditary near-ring-semigroups .

Yoshikazu Katayama, Isomorphisms of the Fourier algebras in crossed

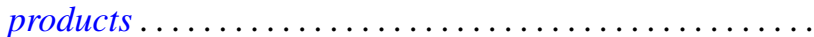

Meir Katchalski and Andrew Chiang-Fung Liu, Symmetric twins and common transversals. .

Mohammad Ahmad Khan, Chain conditions on subgroups of LCA groups ....

Helmut Kröger, Padé approximants on Banach space operator equations ...

Gabriel Michael Miller Obi, An algebraic extension of the Lax-Milgram theorem . .

S. G. Pandit, Differential systems with impulsive perturbation .

Richard Pell, Support point functions and the Loewner variation ...

J. Hyam Rubinstein, Dehn's lemma and handle decompositions of some

4-manifolds

James Eugene Shirey, On the theorem of Helley concerning finite-dimensional subspaces of a dual space.

Oved Shisha, Tchebycheff systems and best partial bases.... 